\title{
Perfil innovador de la industria manufacturera colombiana. Caso del sector metalmecánico de Barranquilla
}

\author{
David Alfredo Ovallos Gazabón* \\ Paola Andrea Amar Sepúlveda*
}

Recibido: 28/11/2013 - Aceptado: 27/06/2014

\begin{abstract}
Resumen
Este artículo presenta los resultados del estudio "Patrones de innovación tecnológica en la industria manufacturera colombiana. El caso del sector metalmecánico de la ciudad de Barranquilla." Se parte de una detallada revisión de la literatura internacional para definir las variables o indicadores del estudio, luego se realizan análisis factoriales y análisis de clúster, que indican la existencia de tres grupos de empresas con patrones de innovación definidos de acuerdo con su grado o nivel de compromiso innovador en alto, medio y reducido, y posteriormente se desarrolla un análisis estructural para definir relaciones de causalidad, con lo que se construye un modelo de análisis y un perfil de empresa manufacturera innovadora. Estos resultados están acompañado de un conjunto de propuestas de estrategias o acciones tendentes al fortalecimiento de las capacidades de las empresas en lo referente a su capacidad y esfuerzo innovador.
\end{abstract}

Palabras clave: perfil innovador, patrones de innovación, competitividad, análisis factorial, análisis de clúster

\footnotetext{
* M. Sc. en Ingeniería. Énfasis en Ingeniería Industrial. Docente Tiempo completo, Ingeniería Industrial, Universidad de la Costa CUC. Calle 58 \# 55 - 66. Barranquilla, Colombia. dovallos1@cuc.edu.co

$2{ }^{* *}$ Doctora en Ingeniería Industrial con énfasis en gestión de la innovación y el conocimiento. Docente Tiempo completo, Administración de empresas, Universidad Tecnológica de Bolívar. Campus Casa Lemaitre. Calle del Bouquet, Carrera 21 \# 25-92. Cartagena de Indias, Colombia. pamar@unitecnologica.edu.co
} 


\title{
Innovative profile of colombian manufacturing industries. Case of metal mechanics sector in barranquilla
}

\begin{abstract}
This article shows the results of the study "Patterns of technological innovation in Colombian manufacturing. The case of Metal Mechanics Sector in Barranquilla." The article begins with a detailed review of the international literature to define the variables or indicators of the study; then it shows the results of a factor and cluster analysis, which indicate the existence of three groups of enterprises with innovation patterns defined according to its degree or level of innovation commitment: high, medium or low. Additionally, it is developed a structural analysis to determine causal relationships between those factors. With the results the authors builds an analysis model and an innovative profile as a model for Colombian manufacturing companies. These results are accompanied by a set of proposed strategies and actions aimed at the enterprises capacities strengthening in terms of their innovative effort.
\end{abstract}

Keywords: Innovative profile, patterns of innovation, competitiveness, factorial analysis, cluster analysis 


\section{INTRODUCCIÓN}

La innovación es vista como factor clave para el desarrollo económico y es posible identificar la existencia de múltiples variables que intervienen en el proceso innovador y que pueden ser combinadas de diferentes maneras, por lo que la identificación de patrones dentro de ese marco es importante para detectar las regularidades que caracterizan los procesos de innovación de diferentes grupos de empresas a fin de mejorar la interpretación del proceso innovador y orientar políticas específicas de apoyo a la actividad innovadora. Para analizar patrones que permitan determinar un perfil de empresa innovadora en un sector empresarial, se hace necesario abordar el estudio de la evolución de la innovación, el cual puede ser realizado en tres niveles: el macro, el meso y el micro, tal como lo plantea [1]. A continuación se hace una breve revisión de los niveles planteados:

\section{- El estudio de la innovación en un nivel macro}

Este nivel de análisis parte de los enfoques de crecimiento neoclásicos basados en la consecución del equilibrio, pasando por el "factor residual" relacionado con el uso más eficiente de los recursos económicos tradicionales de capital y trabajo, hasta los enfoques evolucionistas que destacan la naturaleza dinámica del sistema económico. En este contexto la innovación es vista como un elemento endógeno al señalar que la tecnología se convierte en el resultado de las decisiones de la empresa en materia de conocimiento científico e inversión en $\mathrm{I}++\mathrm{D}$. Posteriormente surge el enfoque de Sistemas de Innovación (SI) que evolucionó hacia los sistemas regionales de innovación, los sistemas sectoriales de innovación y los sistemas tecnológicos de innovación.

\section{- El estudio de la innovación en un nivel meso: Las dinámicas industriales}

La emergencia de conceptos como el de los regímenes tecnológicos puso de manifiesto el carácter dinámico de la evolución industrial y abrió las puertas para que se contemplara el cambio tecnológico como un proceso acumulativo dependiente tanto del conocimiento existente como del comportamiento de los diferentes regímenes. Posteriormente se propuso una taxonomía de modelos sectoriales de cambio tecnológico la cual distinguió diferentes tipos de sectores en función de los resultados de sus actividades de innovación, las fuentes de conocimiento tecnológico que emplean, las exigencias de los clientes y los mecanismos de protección que utilizan para salvaguardar sus innovaciones.

\section{- El estudio de la innovación en un nivel micro: la empresa}

Dentro de este nivel de análisis se destacan inicialmente los modelos lineales tales como el modelo de Impulso o Empuje de la Tecnología o de la Ciencia (Technology 
Push), cuya influencia se extiende desde los años posteriores a la Segunda Guerra Mundial, hasta mediados de los sesenta, y el modelo de tirón de la demanda (demand pull). Como alternativa a los modelos estáticos surgen los primeros modelos de carácter interactivo.

\section{La innovación y su medición}

La importancia y la utilidad de la medición de los procesos de innovación está directamente relacionada con los vínculos existentes y que han sido ampliamente verificados por la evidencia empírica entre innovación, mejoras genuinas de competitividad, crecimiento económico y niveles de bienestar de la sociedad. Los primeros esfuerzos por medir la innovación a través de indicadores estandarizados fueron desarrollados por la Organización para la Cooperación y el Desarrollo Económico (OCDE) y la Comunidad Europea de Naciones, fomentando la discusión sobre la necesidad de preparar el diseño y construcción de indicadores explícitos de la innovación. Para el desarrollo del instrumento de recolección de información utilizado en este trabajo, se empleó el trabajo de [2] y la revisión de otros modelos, lo cual dio origen a un instrumento que incluye los principales indicadores tecnológicos utilizados en la identificación de patrones de innovación y otros que representan un grado de novedad debido a que no son empleados de manera frecuente en la literatura. En este último grupo se destacan elementos tales como la participación de la empresa en un Grupo empresarial, la existencia de cultura orientada a $\mathrm{i}+\mathrm{D}+\mathrm{i}$, la comunicación de la cultura, la actitud frente al fracaso de las actividades de $\mathrm{I}+\mathrm{D}+\mathrm{i}$, la experiencia del equipo de innovación, la existencia de una estrategia de $\mathrm{I}+\mathrm{D}+\mathrm{i}$, la participación en la formulación de la estrategia, la ejecución de la estrategia, el uso de técnicas de gestión de proyectos, la existencia de una política de formación de personal, la existencia de certificación en algún modelo de gestión, la existencia de un modelo de o sistema de gestión del conocimiento y la cooperación con proveedores, entre otros.

\section{- La medición de la innovación en América Latina y Colombia}

Durante la década de los noventa se inició en América Latina un conjunto de esfuerzos e iniciativas con el objeto de medir las actividades tecnológicas y la innovación industrial según [3]. Estas inician con el desarrollo de encuestas de innovación entre 1995 y 1997 en Argentina, Chile, Colombia, México y Venezuela. Posteriormente entre 2000 y 2001, al menos diez países de América Latina realizaron una encuesta de innovación (Argentina, Brasil, Uruguay, Chile, México, Cuba, Ecuador, Panamá, Perú y Trinidad y Tobago) [4]. Si bien se publica y difunde el Manual de Bogotá, no fue posible avanzar en la adopción de un cuestionario común ni en la generación de registros directamente comparables. 
Colombia realizó en 1996 la primera encuesta de desarrollo tecnológico en el establecimiento industrial colombiano (EDT1), a cargo de Colciencias y el Departamento Nacional de Planeación (DNP). La segunda encuesta de desarrollo tecnológico en el establecimiento industrial colombiano (EDT2) se llevó a cabo en 2003 por parte del Observatorio Colombiano de Ciencia y Tecnología (OCyT), Colciencias y el DNP, salvo estos ejercicios puntuales, el país no contaba con indicadores que permitieran caracterizar la dinámica tecnológica de las empresas colombianas. Posteriormente se desarrollaron las siguientes versiones de la Encuesta de Desarrollo e Innovación Tecnológica en la industria manufacturera: EDIT II el año 2005, tomando como período de referencia los años 2003 y 2004; EDIT III en el año 2007 que referencia los períodos 2005-2006; EDIT IV en 2009 que referencia los años 2007-2008 y finalmente la EDIT $\mathrm{V}$ en 2011 que cubre los años 2009-2010.

\section{MATERIALES Y MÉTODOS}

\subsection{Indicadores de innovación y datos}

A partir de la revisión bibliográfica se construyó un instrumento que agrupa un total de 66 variables en seis categorías. Este fue aplicado a 67 empresas del sector metalmecánico de manera aleatoria, considerando que para la ciudad de Barranquilla no existe una base de datos que indique ni por sector empresarial, ni en general para el tejido empresarial de la ciudad su carácter de innovadoras, por lo que este estudio no buscó representatividad estadística, sino más bien, servir de insumo para identificar factores críticos para analizar los procesos de innovación del sector.

\section{- Generalidades de la empresa}

Esta información permite hacer una caracterización general del grupo de estudio y en algunos casos ayuda a explicar el comportamiento de las empresas respecto a la actividad innovadora.

\section{- Innovación y su impacto en la empresa}

Los resultados del proceso innovador han sido estudiados en la literatura especializada, sin que se haya constituido o consolidado un indicador (o conjunto de indicadores) que esté generalmente aceptado [5]. Este apartado incluye la realización de actividades de innovación por parte de la empresa; de igual forma se indaga sobre la percepción de la empresa en relación con su nivel de innovación, y el impacto de las actividades desarrolladas.

\section{- Estrategia y cultura de I+D}

La cultura organizacional se constituye en un factor determinante de innovación, en la medida en que puede obstaculizar o facilitar la innovación [6-9]. 
- Inversión y costos de I+D

Se indaga en este apartado sobre la existencia de un presupuesto específico en la empresa para el desarrollo del ciclo de la innovación, aspecto analizado en el estudio de [3], pero que no se ve integrado en otros trabajos relacionados. Otro aspecto indagado es la inversión en el desarrollo de actividades de innovación (interna, externa, mercadeo de innovaciones, asistencia técnica, consultoría, formación y capacitación, entre otros.) Finalmente este apartado indaga sobre la financiación empleada para las actividades de innovación, aspecto que es común a la mayoría de los estudios ya mencionados.

\section{- Organización para la I+D+i}

Este apartado indaga sobre la relevancia de la estructura organizacional y su relación con el desempeño innovador en las empresas [7, 10]. De igual forma los aspectos relacionados con el papel que juega el talento humano en la innovación de las empresas. Otros aspectos indagados son la flexibilidad organizacional, la existencia de certificaciones bajo algún modelo de gestión, fuentes de conocimiento para la innovación y el uso de herramientas de protección de la propiedad intelectual, las cuales han sido tomadas por diversos autores en la literatura como indicadores de resultado del proceso de innovación [5].

\section{- Relación con el entorno}

La inversión en tecnología y capital humano y en las redes de cooperación juega un papel importante en el desarrollo del proceso innovador de un país, un sector o una industria [11]. La innovación es vista como un proceso que resulta de las diversas interacciones entre los diferentes actores. En este mismo sentido, las redes inter-organizacionales e intersectoriales facilitaron los flujos acelerados de información, recursos y confianza necesarios para la innovación y se han convertido en una estrategia clave, de acuerdo con [11].

\section{RESULTADOS}

\subsection{Análisis para la identificación de patrones de innovación}

En el marco de este trabajo, patrón de innovación hace referencia al grupo de empresas que muestran características similares, en cuanto a las variables definidas, resultados, formas de protección e impactos de las innovaciones realizadas, como así también en lo relativo a las fuentes de información utilizadas y al tipo de interacción con el SNI.

\subsection{Análisis de componentes principales (ACP)}

- Innovación y su impacto en la empresa

La tabla 1 agrupa 16 indicadores, los cuales se concentran en tres factores. El primero de ellos agrupa seis de los indicadores analizados; estos reflejan relación entre las 
diferentes actividades de $\mathrm{I}+\mathrm{D}+\mathrm{i}$ (ACTIDI). El segundo factor se encuentra asociado al impacto de las actividades de $\mathrm{I}+\mathrm{D}+\mathrm{i}$ en la empresa y agrupa un total de ocho indicadores. El tercer factor agrupa las dos variables restantes y se relaciona con el papel de los aspectos organizacionales tales como el tamaño de la empresa y si pertenece a un grupo empresarial en su percepción sobre el nivel de innovación en la empresa (FACTORG).

Tabla 1. Análisis factorial para la categoría Innovación y su impacto en la empresa

\begin{tabular}{|c|c|c|c|c|c|}
\hline Categoría & Denominación & $\begin{array}{l}\text { Eigen } \\
\text { valor }\end{array}$ & $\begin{array}{l}\text { Varianza } \\
\text { explicada }\end{array}$ & $\begin{array}{l}\text { Variables } \\
\text { que incluye }\end{array}$ & Interpretación \\
\hline \multirow{4}{*}{$\begin{array}{l}\text { Innovación } \\
\text { y su im- } \\
\text { pacto en la } \\
\text { empresa }\end{array}$} & ACTIDI & 7.7324 & 51.549 & $\begin{array}{l}\text { BSSMEJ } \\
\text { BSMMN } \\
\text { BSMMI } \\
\text { MPRDMJ } \\
\text { MORGM } \\
\text { TCOMM }\end{array}$ & Actividades de $\mathrm{I}+\mathrm{D}+\mathrm{i}$ \\
\hline & IMPIDI & 2.07472 & 13.831 & $\begin{array}{l}\text { MCALBS } \\
\text { AMPGPS } \\
\text { MPARTM } \\
\text { INGNM } \\
\text { AUMPRD } \\
\text { DISMCP } \\
\text { CUMPREG } \\
\text { APRVRP }\end{array}$ & $\begin{array}{l}\text { Impacto actividades de } \\
\mathrm{I}+\mathrm{D}+\mathrm{i}\end{array}$ \\
\hline & FACTORG & 1.44124 & 9.608 & $\begin{array}{l}\text { TIPEMP } \\
\text { EINNOV }\end{array}$ & Factores Organizacionales \\
\hline & \multicolumn{5}{|c|}{ Varianza total explicada por los tres factores: 74.989} \\
\hline
\end{tabular}

Fuente: elaboración propia

- Estrategia y cultura de I+D +i

En esta categoría se incluyen ocho indicadores agrupados en cuatro factores tal como se muestra en la tabla 2. El primero concentra cuatro de los indicadores analizados que se relacionan con la existencia, conocimiento y actitud de la empresa frente a la innovación (CULID). El segundo factor agrupa tres indicadores asociados con la existencia de una estrategia de $\mathrm{I}+\mathrm{D}+\mathrm{i}$, su elaboración y ejecución de acuerdo con la asignación de recursos como personal, presupuesto, maquinaria y equipos (ESTRA). El tercer factor está relacionado con la apreciación o valoración que se le da a la experiencia del equipo de trabajo para el desarrollo de actividades de $\mathrm{I}+\mathrm{D}+\mathrm{i}$ (EXPER). El cuarto factor está compuesto por la variable que indaga por el uso de técnicas de gestión de proyectos para el desarrollo de las actividades de $\mathrm{I}+\mathrm{D}+\mathrm{i}$ en la empresa (GESPRO). 
Tabla 2. Análisis factorial para la categoría Estrategia y Cultura de I+D+i

\begin{tabular}{|l|l|l|l|l|l|}
\hline \multirow{2}{*}{ Categoría } & \multicolumn{1}{|c|}{ Denominación } & $\begin{array}{c}\text { Eigen } \\
\text { valor }\end{array}$ & $\begin{array}{c}\text { Varianza } \\
\text { explicada }\end{array}$ & $\begin{array}{c}\text { Variables } \\
\text { que incluye }\end{array}$ & \multicolumn{1}{|c|}{ Interpretación } \\
\hline \multirow{2}{*}{$\begin{array}{l}\text { Estrategia y } \\
\text { Cultura de } \\
\text { I+D+i }\end{array}$} & ESTRA & 5.52397 & 69.050 & $\begin{array}{l}\text { CULTINN } \\
\text { COMCULT } \\
\text { FRACACT }\end{array}$ & Cultura de I+D \\
\cline { 2 - 6 } & EXPER & 0.950233 & 11.878 & $\begin{array}{l}\text { ESTDI+D } \\
\text { ELABEST } \\
\text { EJECEST }\end{array}$ & Estrategia \\
\cline { 2 - 6 } & GESPRO & 0.758241 & 9.478 & EXPINNOV & Experiencia para I+D+i \\
\cline { 2 - 6 } & Varianza total explicada por los cuatro factores: 94.059 & Gestión de proyectos \\
\hline
\end{tabular}

Fuente: elaboración propia

- Inversión y costos de I+ D + i

En esta categoría se incluyen dieciséis indicadores y está compuesta por tres factores que se presentan en la tabla 3 que agrupan cerca del $64 \%$ de la varianza total de la categoría. El primero agrupa ocho indicadores relacionados con el tipo de actividades de $\mathrm{I}+\mathrm{D}+\mathrm{i}$ en las cuales la empresa ha invertido (ACTIV). El segundo factor se relaciona con la fuente de financiación que emplea la empresa para costear la realización de las actividades de $\mathrm{I}+\mathrm{D}+\mathrm{i}$ (FUEFIN). El tercer factor está compuesto por la variable que indaga sobre la existencia de un presupuesto específico para el desarrollo de actividades de $\mathrm{I}+\mathrm{D}+\mathrm{i}$ en la empresa.

Tabla 3. Análisis factorial para la categoría Inversión y costos de I+D+i

\begin{tabular}{|l|l|l|l|l|l|}
\hline \multicolumn{1}{|c|}{ Categoría } & Denominación & $\begin{array}{c}\text { Eigen } \\
\text { valor }\end{array}$ & $\begin{array}{c}\text { Varianza } \\
\text { explicada }\end{array}$ & $\begin{array}{l}\text { Variables que } \\
\text { incluye }\end{array}$ & Interpretación \\
\hline & & & $\begin{array}{l}\text { INVSI+DI } \\
\text { INVSI+DE } \\
\text { INVSTIC }\end{array}$ & \\
Inversión \\
$\begin{array}{l}\text { y costos de } \\
\text { I+D+i }+ \text { ACTIV }\end{array}$ & 6.7255 & 42.034 & $\begin{array}{l}\text { INVSI } \\
\text { INVSTT } \\
\text { INVSATC } \\
\text { INVSIDI } \\
\text { INVSFYC }\end{array}$ & Actividades de I+D+i \\
& & & & & \\
\hline
\end{tabular}


Perfil innovador de la industria manufacturera colombiana. Caso del sector metalmecánico de Barranquilla 123

\begin{tabular}{|l|l|l|l|l|l|}
\hline Categoría & Denominación & $\begin{array}{c}\text { Eigen } \\
\text { valor }\end{array}$ & $\begin{array}{c}\text { Varianza } \\
\text { explicada }\end{array}$ & $\begin{array}{l}\text { Variables que } \\
\text { incluye }\end{array}$ & Interpretación \\
\hline & FUEFIN & 2.2136 & 13.835 & $\begin{array}{l}\text { FINRECP } \\
\text { FINROEG } \\
\text { FINRPUB } \\
\text { FINRBPR } \\
\text { FINOEMP } \\
\text { FINRCAP } \\
\text { FINRCOOP }\end{array}$ & Fuentes de Financiación \\
\hline & PRESU & 1.37334 & 8.583 & PPTOINN & $\begin{array}{l}\text { Presupuesto para actividades } \\
\text { de I+D+i }\end{array}$ \\
\hline
\end{tabular}

Fuente: elaboración propia

- Organización para la I+D + i

En esta categoría se incluyen dieciséis indicadores compuestos por siete factores que agrupan cerca del $90 \%$ de la varianza total de la categoría y se presentan en la tabla 4. El primer factor agrupa tres indicadores relacionados con la existencia de una política empresarial para la formación y/o actualización del personal en temas de $\mathrm{I}+\mathrm{D}+\mathrm{i}$ ya sea mediante actividades de formación o a través de la asistencia a seminarios, ferias u otro tipo de eventos de divulgación científica, tecnológica o comercial (FORMA). El segundo factor agrupa un total de cinco indicadores relacionados con la generación y/o adquisición y gestión del conocimiento en la empresa (GESTI). El tercer factor está relacionado con la existencia de un departamento o área específica para el desarrollo de las actividades de $\mathrm{I}+\mathrm{D}+\mathrm{i}$ (DEPID). El cuarto factor agrupa cuatro indicadores afines a la identificación del área de la empresa en la cual se llevan a cabo las acciones de $\mathrm{I}+\mathrm{D}+\mathrm{i}$ (LUGID). El quinto factor está relacionado con el grado o nivel de flexibilidad que tiene la empresa para promover el desarrollo de actividades de I+D+i (FLEXI). El sexto factor se relaciona con la existencia de modelos de gestión en la empresa y su relación con la innovación (MODGEST) y el séptimo factor hace referencia al uso de herramientas de protección de la propiedad intelectual derivada del desarrollo de actividades de $\mathrm{I}+\mathrm{D}+\mathrm{i}$ (PROTEPI).

\section{- Relación con el entorno}

Esta categoría incluye diez indicadores y está compuesta por dos factores, los cuales explican cerca del $62 \%$ de la varianza total de la categoría que se presentan en la tabla 5. El primer factor agrupa nueve indicadores relacionados con la relevancia que le otorga la empresa a las actividades de cooperación para $\mathrm{I}+\mathrm{D}+\mathrm{i}$ y los actores del SIN con los cuales coopera o ha cooperado (ACTCOOPE). El segundo factor está relacionado con 
la realización de ejercicio de vigilancia tecnológica como apoyo a la innovación en la empresa (VIGILTEC).

Tabla 4. Análisis factorial para la categoría Organización para la I+D+i

\begin{tabular}{|c|c|c|c|c|c|}
\hline Categoría & $\begin{array}{l}\text { Denomina- } \\
\text { ción }\end{array}$ & $\begin{array}{l}\text { Eigen } \\
\text { valor }\end{array}$ & $\begin{array}{l}\text { Varianza } \\
\text { explicada }\end{array}$ & $\begin{array}{c}\text { Variables que } \\
\text { incluye }\end{array}$ & Interpretación \\
\hline \multirow{8}{*}{$\begin{array}{l}\text { Organiza- } \\
\text { ción para la } \\
\mathrm{I}+\mathrm{D}+\mathrm{i}\end{array}$} & FORMA & 8.6516 & 54.073 & $\begin{array}{l}\text { POLFORM } \\
\text { PERSCAPI+D } \\
\text { ASISFEI+D }\end{array}$ & $\begin{array}{l}\text { Formación del personal en } \\
I+D+i\end{array}$ \\
\hline & GESTI & 1.4926 & 9.329 & $\begin{array}{l}\text { GESTCON } \\
\text { FCONINT } \\
\text { FCONMERC } \\
\text { FCONFINST } \\
\text { FCONOFTES } \\
\end{array}$ & $\begin{array}{l}\text { Gestión y fuentes de } \\
\text { conocimiento }\end{array}$ \\
\hline & DEPID & 1.2649 & 7.906 & $\mathrm{DPTOI}+\mathrm{D}$ & Departamento de I+D \\
\hline & LUGID & 0.9615 & 6.010 & $\begin{array}{l}\text { ACTI+DDPTO } \\
\text { ACTI+DPROD } \\
\text { ACTI+DCAL } \\
\text { ACTI+DOA }\end{array}$ & $\begin{array}{l}\text { Lugar donde se desarrollan las } \\
\text { actividades de I+D }\end{array}$ \\
\hline & FLEXI & 0.7287 & 4.555 & CAMBORG & $\begin{array}{l}\text { Flexibilidad organizacional } \\
\text { para la } \mathrm{I}+\mathrm{D}+\mathrm{i}\end{array}$ \\
\hline & MODGEST & 0.6952 & 4.346 & MODGEST & $\begin{array}{l}\text { La empresa se encuentra certi- } \\
\text { ficada bajo uno o más modelos } \\
\text { de gestión }\end{array}$ \\
\hline & PROTEPI & 0.5585 & 3.491 & PROTPI & $\begin{array}{l}\text { Protección de la propiedad } \\
\text { intelectual }\end{array}$ \\
\hline & \multicolumn{5}{|c|}{ Varianza total explicada por los cuatro factores: 89.709} \\
\hline
\end{tabular}

Fuente: elaboración propia

Tabla 5. Análisis factorial para la categoría Relación con el entorno

\begin{tabular}{|l|l|l|l|l|l|}
\hline Categoría & Denominación & $\begin{array}{c}\text { Eigen } \\
\text { valor }\end{array}$ & $\begin{array}{c}\text { Varianza } \\
\text { explicada }\end{array}$ & $\begin{array}{l}\text { Variables que } \\
\text { incluye }\end{array}$ & Interpretación \\
\hline & & & & $\begin{array}{l}\text { COOPI+D } \\
\text { COOPOEG } \\
\text { COOPPOV } \\
\text { COOPCLTES } \\
\text { el entorno }\end{array}$ \\
& ACTCOOPE & 4.89785 & 48.978 & $\begin{array}{l}\text { COOPCOMP } \\
\text { COOPCLAB } \\
\text { COOPUNIV } \\
\text { COOPOPINV } \\
\text { COOPCTEC }\end{array}$ & Actividades de cooperación \\
\hline & VIGILTEC & 1.29996 & 13.000 & ACTVIGTEC & Vigilancia tecnológica \\
\hline & Varianza total explicada por los cuatro factores: 61.978 \\
\hline
\end{tabular}




\subsection{Análisis de clúster: patrones de innovación}

El paso siguiente consistió en la realización de un análisis de clúster con base en los factores descritos. Para esto se empleó el procedimiento de Ward y la medida de distancia euclidiana. Como resultado se obtienen tres patrones con características diferenciales en los aspectos recogidos en los factores. La figura 1 presenta el resultado de este análisis.

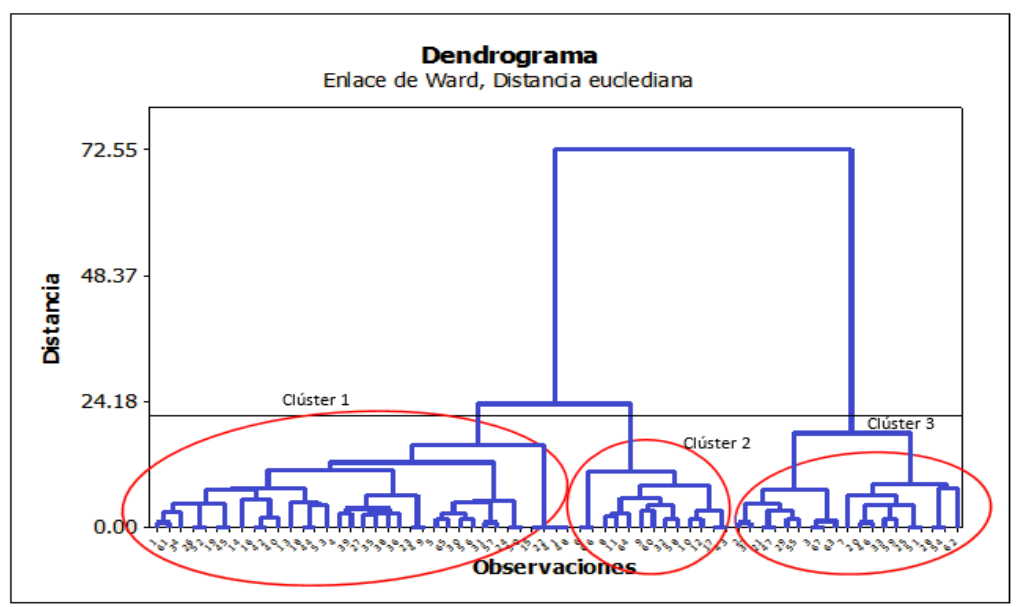

Figura 1. Dendograma para los factores estudiados

Fuente: Salida software estadístico Minitab ${ }^{\oplus} 16$

A fin de facilitar la interpretación de los resultados se presenta la información en términos relativos usando el método empleado por $[2,12]$. Los símbolos "+", "=" ó "-", reflejan el posicionamiento relativo de cada patrón en cada uno de los factores con respecto al promedio de la base. La tabla 6 muestra el comportamiento de los factores analizados.

Tabla 6. Grado de compromiso y perfil innovador de los patrones

\begin{tabular}{|l|c|c|c|}
\hline \multicolumn{1}{|c|}{ Variable } & Clúster 2 & Clúster 1 & Clúster 3 \\
\hline Innovación y su impacto en la empresa & & & \\
\hline - FACTORG & + & $=$ & - \\
\hline - ACTIDI & + & $=$ & - \\
\hline - IMPIDI & + & & - \\
\hline Estrategia y cultura para la I+D+i & & $=$ & - \\
\hline - CULID & + & $=$ & - \\
\hline - EXPINNOV & + & & \\
\hline
\end{tabular}




\begin{tabular}{|c|c|c|c|}
\hline Variable & Clúster 2 & Clúster 1 & Clúster 3 \\
\hline - ESTRA & ++ & - & - \\
\hline - GESTPRY & ++ & - & -- \\
\hline \multicolumn{4}{|l|}{ Inversión y costos de la $\mathrm{I}+\mathrm{D}+\mathrm{i}$} \\
\hline - PPTOINN & ++ & - & - \\
\hline - ACTIV & + & $=$ & - \\
\hline - FUEFIN & + & $=$ & - \\
\hline \multicolumn{4}{|l|}{ Organización para $\mathrm{I}+\mathrm{D}+\mathrm{i}$} \\
\hline - DPTOI+D & ++ & $=$ & -- \\
\hline - LUGID & + & $=$ & - \\
\hline - FORMA & + & $=$ & - \\
\hline - CAMBORG & ++ & $=$ & - \\
\hline - MODGEST & + & $=$ & -- \\
\hline - GESTI & + & $=$ & - \\
\hline - PROTPI & ++ & - & - \\
\hline \multicolumn{4}{|l|}{ Relación con el entorno } \\
\hline - ACTCOOPE & + & $=$ & - \\
\hline - ACTVIGTEC & ++ & - & -- \\
\hline Compromiso innovador & Muy elevado & Medio & Reducido \\
\hline
\end{tabular}

Fuente: [13]

Si se tienen en cuenta todas las categorías de indicadores, se puede apreciar que existe una clara diferenciación entre los tres clústers/patrones definidos. De acuerdo con lo que se llamará en adelante el compromiso innovador de las empresas, que no es más que la sumatoria de esfuerzos encaminados a fortalecer el desarrollo de actividades de $\mathrm{I}+\mathrm{D}+\mathrm{i}$. Al respecto, es posible identificar un MUY ELEVADO compromiso innovador en el clúster 2, mientras que el clúster 1 muestra un nivel MEDIO de compromiso innovador, y el clúster 3 muestra un REDUCIDO compromiso innovador.

- Patrón 1. Empresas con elevado compromiso innovador

La principal característica de las empresa agrupadas en el clúster 2 es su elevado compromiso innovador y que se encuentran orientadas al desarrollo de capacidades 
internas para la realización de actividades de $\mathrm{I}+\mathrm{D}+\mathrm{i}$, lo cual se ve reflejado en el alto nivel alcanzado por los aspectos relacionados con el desarrollo y ejecución de una estrategia de fomento a la I+D+i (ESTRA), lo que supone la existencia de los recursos y medios para financiar dichas actividades y se confirma con el nivel alcanzado por la variable de existencia de un presupuesto específico (PPTOINN). Este grupo de empresas se caracteriza por tener como una de sus principales fuentes de información para el desarrollo de las innovaciones al entorno a través del desarrollo de continuos ejercicios de Vigilancia Tecnológica (ACTVIGTEC). Esta frecuente búsqueda de información actualizada le permite a la empresa adaptarse a los cambios del entorno, por lo cual estas empresas poseen un alto nivel en lo relacionado con el cambio o capacidad de modificar la estructura organizacional para favorecer la innovación (CAMBORG).

\section{- Patrón 2. Empresas con un compromiso innovador medio}

Las empresas del siguiente clúster se caracterizan por tener un compromiso medio de innovación en el marco del esfuerzo tanto monetario como administrativo y organizacional para el desarrollo de las actividades de $\mathrm{I}+\mathrm{D}+\mathrm{i}$. Se podría afirmar que es el grupo que representa la actualidad del sector metalmecánico en la ciudad de Barranquilla, ya que son pocas empresas las que se destacan por su compromiso y esfuerzo innovador (especialmente empresas grandes), mientras que cerca del $90 \%$ del tejido empresarial de la ciudad está constituido por empresas medianas y pymes, las cuales tienen una actividad "promedio" en lo que respecta a aspectos organizacionales (FACTORG), desarrollo de actividades de $\mathrm{I}+\mathrm{D}+\mathrm{i}$, su impacto (IMPIDI), Cultura para la $\mathrm{I}+\mathrm{D}+\mathrm{i}(\mathrm{CULID})$, el valor de la experiencia del equipo para el proceso de innovación (EXPINNOV), actividades de cooperación (ACTCOOPE). Respecto al resto de factores analizados, estas empresas se encuentran por debajo del promedio.

\section{- Patrón 3. Empresas con un compromiso innovador reducido}

Las empresas del tercer clúster muestran un muy reducido compromiso innovador que se refleja especialmente en la realización del gasto más reducido y menos regular de todos los patrones, pero también en el fuerte aislamiento que mantienen con relación a los demás agentes del SNI. Es posible observar que existe una marcada tendencia a desarrollar muy pocas o ninguna actividad de $\mathrm{I}+\mathrm{D}+\mathrm{i}$, las principales fuentes que utilizan para innovar son las internas, a través de gastos que orientan preferente a actividades de I+D y cierta presencia de recursos humanos en tales actividades. En este tipo de empresas las pocas actividades de innovación se encuentran orientadas casi exclusivamente a la innovación en productos, aunque con escaso grado de novedad. En síntesis, se trata de empresas que escasamente innovan en productos buscando ampliar la gama de los mismos a través de esfuerzos propios, reducidos e irregulares, destinados a I+D y en el marco de un casi total aislamiento del resto del SNI. 


\subsection{Análisis estructural}

Mediante la utilización de matrices y gráficos de relación, se trató de interconectar todas las variables en estudio para así detectar aquellas claves que ejercen la mayor influencia sobre las restantes. Como resultado se obtiene un modelo preliminar que da información acerca de las relaciones de motricidad y dependencia entre las principales variables del modelo. La figura 2 presenta el modelo preliminar que se construye a partir del análisis.

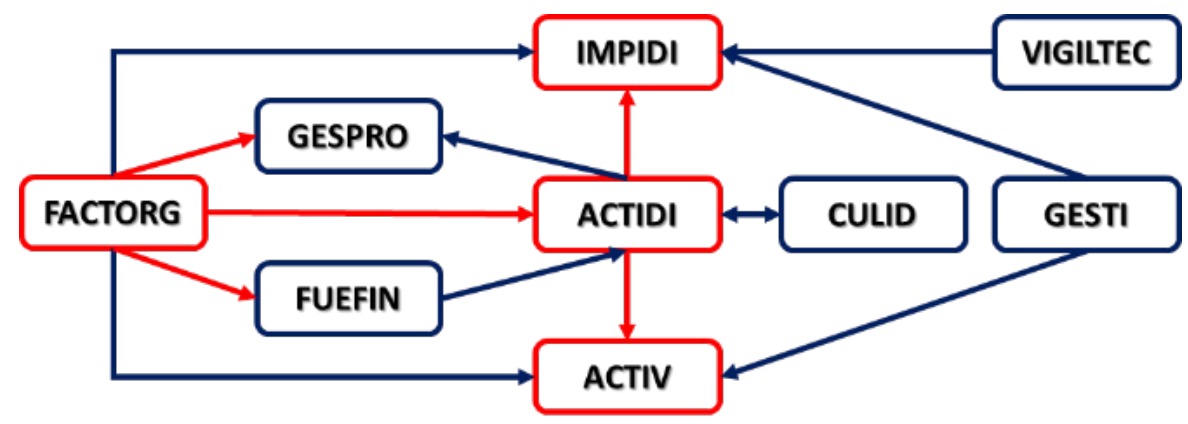

Figura 2. Relaciones entre las variables clave del sistema

Fuente: [13]

Este ejercicio permitió identificar relaciones de causalidad entre variables, relaciones que posteriormente sirvieron de insumo para la generación de lineamientos para diseñar estrategias orientadas a dinamizar el comportamiento innovador de la industria manufacturera colombiana con el fin de mejorar su capacidad de respuesta a las exigencias del entorno e incrementar su competitividad, teniendo como caso de estudio al sector metalmecánico de la ciudad de Barranquilla.

\section{MODELO DE ANÁLISIS PROPUESTO}

El modelo de análisis (figura 3) que se plantea consta de cuatro áreas o conjunto de elementos que a consideración del autor, y basado en la revisión de literatura y de los resultados obtenidos con anterioridad, condicionan el comportamiento innovador de una empresa y su impacto en la misma y en el entorno. Estas áreas o elementos se refieren a ámbitos de la empresa y cada uno de ellos está formado por elementos cuya existencia formal o informal es precisa para que la innovación exista. Su nivel de formalidad, los recursos implicados y el grado de compromiso con ellos, asumido por la organización, pueden ser considerados como indicadores de su capacidad innovadora. 


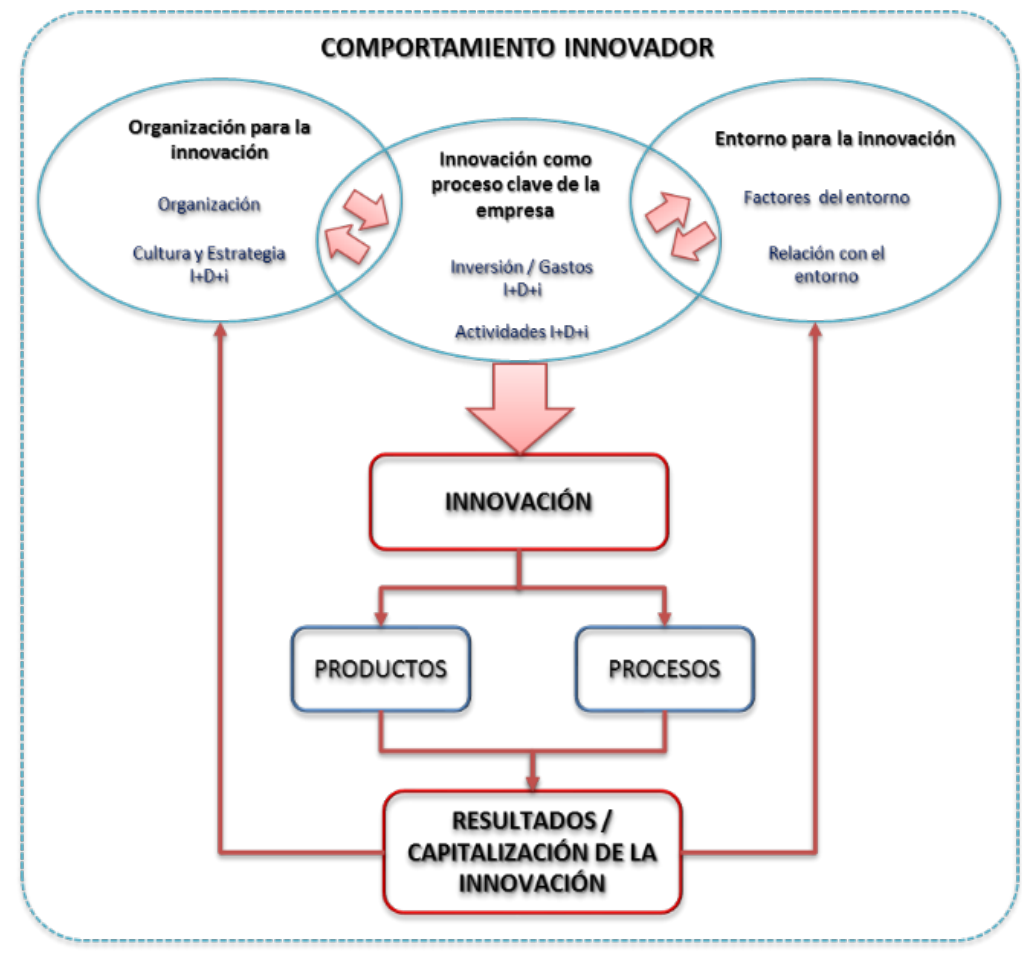

Figura 3. Modelo de análisis propuesto

Fuente: [13]

\section{- Organización para la innovación}

Para el desarrollo de procesos de innovación, es necesario contar con elementos básicos que aseguren que la empresa esté en permanente actitud innovadora. La empresa requiere de una estructura organizacional que facilite el desarrollo del proceso innovador y es capaz de adaptarse a los cambios requeridos por el proceso [10, 14]. De igual forma al hablar de elementos básicos para la innovación se espera que la empresa cuente con un área, personal altamente capacitado, políticas de formación, y dedicación formal al desarrollo de actividades de $\mathrm{I}+\mathrm{D}+\mathrm{i}$, cuyos frutos deberán ser cubiertos por herramientas de protección de la propiedad intelectual a fin de garantizar su aprovechamiento en el largo plazo. Otro elemento relevante dentro del modelo es la cultura organizacional en la medida en que puede obstaculizar o facilitar la innovación y la existencia de una estrategia definida de $\mathrm{I}+\mathrm{D}+\mathrm{i}$ en la empresa de acuerdo a $[6,7$, $10,14,15]$ entre otros. En este contexto se espera que las empresas evolucionen hacia organizaciones ambidiestras tal como las describen [16]. 
- Entorno para la innovación

El modelo de análisis propuesto incorpora la importancia del entorno como elemento condicionante. En tal sentido, las actuaciones de los agentes del entorno (competidores, clientes, proveedores, gobiernos, institutos de investigación, etc.) condicionan las actividades de innovación de la empresa y ofrecen oportunidades de colaboración fundamentales. De esta manera, el modelo de innovación es dinámico, ya que el entorno cambia y también cambian las estrategias y actividades de innovación de la empresa.

- Innovación como proceso clave de la empresa

La innovación debe ser una operación empresarial [17]. Debe ser considerada como un proceso clave de la empresa y debe estar incluida de manera transversal en cada uno de los procesos y actividades que hacen parte de la organización. Para que la innovación sea vista de esta forma, es necesario que existan medios y personas dedicados a ella de manera formal, que existan procesos definidos y que se cuente con un mínimo de herramientas para el desarrollo de las actividades que requiere el proceso de innovación. De esta manera se garantiza que los procesos innovadores sean interiorizados como cualquier otra operación y por lo tanto estén sometidos a métricas adecuadas a su gestión.

\section{- Resultados y capitalización de la innovación}

Una innovación sostenida solo es posible si existe el convencimiento constante en la empresa de que el conjunto de las innovaciones proporciona beneficios. Por tanto se debe contar con una permanente y clara conciencia de que se está aportando valor mediante este arriesgado proceso, y por esto se debe evaluar la mejora en los productos y servicios ofrecidos, en la eficiencia interna de los procesos y en la capitalización de los resultados de las innovaciones, a través de la generación de derechos de propiedad industrial e intelectual o mejora de imagen. El modelo contempla ese retorno de información o beneficios de la innovación tanto al entorno, como a la misma organización.

\section{PERFIL DE LA INDUSTRIA MANUFACTURERA COLOMBIANA INNOVADORA. EL CASO DEL SECTOR METALMECÁNICO DE LA CIUDAD DE BARRANQUILLA}

En la tabla 7 se presenta un listado de las características deseables para las empresas del sector metalmecánico de la ciudad de Barranquilla, las cuales, de ser alcanzadas en los niveles propuestos, tenderían al mejoramiento de las actividades de innovación de las empresas del sector. 
Tabla 7. Perfil empresa innovadora

\begin{tabular}{|c|c|}
\hline característica & Nivel empresa innovadora \\
\hline Estrategia de $\mathrm{I}+\mathrm{D}+\mathrm{i}$ & $\begin{array}{l}\text { Estrategia de } \mathrm{I}+\mathrm{D}+\mathrm{i} \text { definida y ampliamente conocida por toda la } \\
\text { organización con recursos asignados para su ejecución. }\end{array}$ \\
\hline Visión de la innovación & $\begin{array}{l}\text { Innovación vista como un medio para alcanzar los objetivos estra- } \\
\text { tégicos de la empresa. }\end{array}$ \\
\hline Ideas innovadoras & $\begin{array}{l}\text { La empresa promueve, sistematiza y emplea las ideas generadas } \\
\text { por sus empleados. }\end{array}$ \\
\hline Actitud frente al fracaso & $\begin{array}{l}\text { La empresa les otorga a los empleados la libertad y el estímulo } \\
\text { para que fracasen sin que ello signifique poner en peligro sus } \\
\text { carreras }\end{array}$ \\
\hline Enfoque de proyectos & $\begin{array}{l}\text { La empresa usa el enfoque de proyectos para el desarrollo de } \\
\text { proyectos de innovación. }\end{array}$ \\
\hline $\begin{array}{l}\text { Procesos de innovación } \\
\text { estructurados }\end{array}$ & $\begin{array}{l}\text { La empresa tiene estandarizados todos los procesos comunes para } \\
\text { el desarrollo de proyectos de innovación }\end{array}$ \\
\hline $\begin{array}{l}\text { Identificación de personal clave para } \\
\text { el desarrollo de la innovación }\end{array}$ & $\begin{array}{l}\text { La empresa dispone de una base de datos con capacidades y talen- } \\
\text { tos del personal para el desarrollo de proyectos de } \mathrm{I}+\mathrm{D}+\mathrm{i}\end{array}$ \\
\hline Sistema de incentivos & $\begin{array}{l}\text { La empresa tiene definido un sistema de incentivos para el desa- } \\
\text { rrollo de proyectos de } \mathrm{I}+\mathrm{D}+\mathrm{i}\end{array}$ \\
\hline $\begin{array}{l}\text { Compromiso de la dirección con la } \\
\mathrm{I}+\mathrm{D}+\mathrm{i}\end{array}$ & $\begin{array}{l}\text { En la empresa la alta dirección se encuentra comprometida con el } \\
\text { desarrollo de proyectos de innovación }\end{array}$ \\
\hline $\begin{array}{l}\text { Anticipación a las tendencias del } \\
\text { mercado }\end{array}$ & $\begin{array}{l}\text { La empresa realiza actividades periódicas de vigilancia } \\
\text { tecnológica }\end{array}$ \\
\hline $\begin{array}{l}\text { Procesamiento y asimilación de la } \\
\text { información }\end{array}$ & $\begin{array}{l}\text { La empresa ha establecido procesos para el procesamiento y } \\
\text { asimilación de la información proveniente del entorno }\end{array}$ \\
\hline Cooperación interna y externa & $\begin{array}{l}\text { Existen redes de cooperación para el desarrollo de proyectos de } \\
\text { innovación }\end{array}$ \\
\hline $\begin{array}{l}\text { Política de formación y actualiza- } \\
\text { ción del personal }\end{array}$ & $\begin{array}{l}\text { La empresa posee una política definida de formación y actualiza- } \\
\text { ción del personal }\end{array}$ \\
\hline Cultura de $\mathrm{I}+\mathrm{D}+\mathrm{i}$ & $\begin{array}{l}\text { Existe una cultura orientada a la } \mathrm{I}+\mathrm{D}+\mathrm{i} \text { en la empresa amplia- } \\
\text { mente comunicada y aceptada por el personal }\end{array}$ \\
\hline Departamento o área de $\mathrm{I}+\mathrm{D}+\mathrm{i}$ & $\begin{array}{l}\text { La empresa posee un área específica para el desarrollo de proyec- } \\
\text { tos de } \mathrm{I}+\mathrm{D}+\mathrm{i}\end{array}$ \\
\hline
\end{tabular}




\begin{tabular}{|l|l|}
\hline \multicolumn{1}{|c|}{ característica } & \multicolumn{1}{c|}{ Nivel empresa innovadora } \\
\hline $\begin{array}{l}\text { Personal dedicado a actividades de } \\
\mathrm{I}+\mathrm{D}+\mathrm{i}\end{array}$ & $\begin{array}{l}\text { La empresa dispone de personal asignado de manera formal al } \\
\text { desarrollo de proyectos de } \mathrm{I}+\mathrm{D}+\mathrm{i}\end{array}$ \\
\hline Presupuesto específico para $\mathrm{I}+\mathrm{D}+\mathrm{i}$ & $\begin{array}{l}\text { La empresa dispone de un presupuesto específico para el desarro- } \\
\text { llo de proyectos de } \mathrm{I}+\mathrm{D}+\mathrm{i}\end{array}$ \\
\hline Actividades de $\mathrm{I}+\mathrm{D}+\mathrm{i}$ & La empresa realiza actividades de I+D+i \\
\hline $\begin{array}{l}\text { Financiación de actividades de } \\
\mathrm{i}+\mathrm{D}+\mathrm{i} \text { con otros recursos (no solo } \\
\text { propios) }\end{array}$ & $\begin{array}{l}\text { La empresa financia el desarrollo de proyectos de innovación con } \\
\text { recursos públicos, recursos de contrapartidas, donaciones, etc. }\end{array}$ \\
\hline
\end{tabular}

Fuente: [13]

\section{PROPUESTA DE LINEAMIENTOS Y ESTRATEGIAS PARA EL SECTOR METALMECÁNICO DE LA CIUDAD DE BARRANQUILLA}

\subsection{Organización para la innovación}

Se presenta un conjunto de acciones propuestas que apuntan al desarrollo de una organización orientada a la innovación, apoyada en una cultura innovadora y operacionalizada en una estrategia de $\mathrm{I}+\mathrm{D}+\mathrm{i}$ con alcance, recursos involucrados y responsabilidades definidas, y comunicada en toda la empresa.

- Establecer una estrategia o proceso formal para la innovación en la empresa

- Desarrollo de campañas masivas de ideas

- Estructuración de grupos de investigación en áreas de I+D+i en las empresas del sector

- Diseño de programas de gestión estratégica de $\mathrm{I}+\mathrm{D}+\mathrm{i}$ en áreas como: nuevos materiales, fuentes alternativas de energía, manufactura, soldadura, técnicas de mecanizado, automatización con alto impacto en la cadena productiva metalmecánica.

- Promoción del conocimiento y apropiación en materia de propiedad intelectual en las empresas de la cadena metalmecánica

\subsection{Entorno para la innovación}

Estas acciones apuntan al desarrollo de una Organización con un alto grado de interrelación con el entorno en que operan a través de actividades de cooperación en proyectos de $\mathrm{I}+\mathrm{D}+\mathrm{i}$. 
- Diseño de programas de asistencia técnica de la grande empresa hacia las medianas y pequeñas empresas de baja complejidad tecnológica, en el proceso de transformación de la cadena

- Aprovechamiento de convocatorias y otras oportunidades de financiación de proyectos de $\mathrm{I}+\mathrm{D}+\mathrm{i}$ mediante el establecimiento de acuerdos entre empresas y CDT y universidades

- Programas de integración competitiva entre pequeñas y grandes empresas

- Construcción de redes nacionales e internacionales de conocimiento en $\mathrm{I}+\mathrm{D}+\mathrm{i}$

- Identificación de las tendencias tecnológicas, plataformas y mercados asociados al desarrollo del sector

- Foro de Integración e Intercambio Tecnológico del Sector Metalmecánico de La Región Caribe.

- Red de Intercambio de Capital Intelectual.

\subsection{Innovación como proceso clave de la empresa}

Según lo señalado por [17] la innovación debe ser considerada como un proceso clave de la empresa, y debe estar incluida de manera transversal en cada uno de los procesos y actividades que hacen parte de la organización. La tabla 9 presenta las acciones propuestas para tal fin.

- El enfoque de proyectos permite priorizar las iniciativas de I+D+i de la empresa; de igual forma clarifica la asignación de recursos y permite hacer un seguimiento puntual a cada iniciativa. Las empresas altamente innovadoras se enfocan en ideas priorizadas y las desarrollan bajo el enfoque de proyectos (PMI).

- Programa para la certificación internacional en gestión de proyectos del PMI (Project Management Institute)

- Sensibilización y compromiso de la alta dirección

- Sistema de gestión de la innovación

\section{CONCLUSIONES Y TRABAJOS FUTUROS}

Para identificar los patrones de innovación de la empresa metalmecánica de la ciudad de Barranquilla se empleó un abordaje estadístico utilizando dos técnicas. En primer lugar, para reducir la dimensión del fenómeno, se efectuó un conjunto de análisis factoriales que permitieron identificar las variables que mayor contribución hacen a 
explicar su variabilidad. Luego, a partir de los factores obtenidos se realizó un análisis de clúster que permitió agrupar a las empresas en conjuntos homogéneos en términos de su comportamiento innovador. Adicionalmente se llevó a cabo un análisis estructural a fin de identificar y describir las relaciones existentes entre las variables o "factores de cambio" que caracterizan el sistema de estudio.

Al hacer el análisis de clúster se obtuvieron tres aglomeraciones o grupos de empresas con características definidas de acuerdo con los factores antes mencionados. Esto permitió diferenciar el compromiso innovador de las empresas, que no es más que la sumatoria de esfuerzos encaminados a fortalecer el desarrollo de actividades de $\mathrm{I}+\mathrm{D}+\mathrm{i}$. Fue posible identificar un MUY ELEVADO compromiso innovador en el clúster 2, mientras que el clúster 1 muestra un nivel MEDIO de compromiso innovador y el clúster 3 muestra un REDUCIDO compromiso innovador.

Las principales características del perfil innovador identificado están relacionadas con la existencia de una estrategia única y relevante de $\mathrm{I}+\mathrm{D}+\mathrm{i}$ que es conocida por todas las personas que están involucradas con el desenvolvimiento de la empresa. En lo relacionado con los aspectos organizacionales tales como el tamaño o el mercado en que se mueve, la literatura y la evidencia empírica señala que una organización innovadora puede ser pequeña o no conocida globalmente, pero ser altamente innovadora. Si una empresa no tiene una estrategia definida, única, no va será innovadora según lo plantea [7]. Las empresas innovadoras visualizan la innovación no como el fin del esfuerzo de la organización, sino como un medio para alcanzar los objetivos estratégicos de la empresa. Por otra parte, son empresas en las cuales desde la alta dirección se promueven, sistematizan y emplean las ideas generadas por el personal en proyectos de innovación, las cuales se realizan bajo una estructura de recursos definida para tal fin. Adicionalmente son empresas que cooperan con otros elementos del entorno para el desarrollo de actividades de innovación que van desde la búsqueda de información, hasta el desarrollo de proyectos de innovación conjunto, las cuales son financiadas con recursos de diversas fuentes, en especial de recursos públicos para la investigación y de recursos de cooperación internacional.

El ejercicio presenta la limitación de ser un estudio de tipo exploratorio-descriptivo, por lo que si bien se generan resultados que aportan al mejoramiento del conocimiento sobre las características del proceso de innovación tecnológica en la industria manufacturera colombiana teniendo como objeto de estudio al sector metalmecánico de la ciudad de Barranquilla, no se tuvieron en cuenta aspectos relevantes como el monto de inversión en actividades de $\mathrm{I}+\mathrm{D}+\mathrm{i}$ ni su resultado también expresado en dinero debido a la sensibilidad de esta información por parte de las empresas. No fue posible plantear una relación entre el compromiso innovador y el desempeño económico de las 
empresas. Por otra parte, el ejercicio indagó sobre actividades y resultados de $\mathrm{I}+\mathrm{D}+\mathrm{i}$ en los últimos dos años; en este caso, también por la poca información que posee la mayoría de las empresas sobre proyectos de innovación llevados a cabo en un período mayor, se plantea la posibilidad de ampliar el estudio teniendo como base un periodo de tiempo más amplio.

Otro aspecto para tener en cuenta en futuras investigaciones es el estudio del comportamiento de otros sectores de manera individual o comparativa usando la metodología planteada en este trabajo a fin de tener resultados comparables. Por otra parte el estudio dio mayor relevancia a la innovación tecnológica y no a la innovación como un todo, por lo que se puede ampliar el horizonte de análisis incluyendo y dando mayor relevancia a la innovación en gestión o innovación organizacional, aspecto que fue analizado en menor grado en este estudio dado el objetivo de la investigación orientado principalmente a la innovación tecnológica en las empresas.

\section{REFERENCIAS}

[1] J. Vega Jurado, «Las estrategias de innovación en la industria manufacturera española: sus determinantes y efectos sobre el desempeño innovador.,» INGENIO, Valencia, España, 2008.

[2] D. Milesi, Patrones de innovación en la industria manufacturera argentina, Buenos Aires, 2006.

[3] F. Malaver Rodríguez y M. Vargas Pérez, «Hacia una caracterización de los procesos de innovación en la industria colombiana. Los resultados de un estudio de casos,» Revista Latinoamericana de Administración, vol. I, n. ${ }^{\circ}$ 33, pp. 5-33, 2004.

[4] D. Olaya y F. Peirano, «El camino recorrido por América Latina en el desarrollo de indicadores para la medición de la sociedad de la información y la innovación tecnológica,» Revista Iberoamericana de Ciencia Tecnologia y Sociedad, vol. 3, n. ${ }^{\circ}$ 9, pp. 153-164, 2007.

[5] C. Camisón y A. Villar, «Un análisis del proceso innovador de las empresas valencianas: objetivos, estrategias y resultados.,» de Decisiones basadas en el conocimiento y en el papel social de la empresa: XX Congreso anual de AEDEM (p. 39), 2007.

[6] G. Calderón Hernández y J. C. Naranjo Valencia, «Perfil cultural de las empresas innovadoras. Un estudio de caso en empresas metalmecánicas,» Cuadernos de Administración, vol. 20, n. ${ }^{\circ}$ 34, pp. 161-189, 2007.

[7] M. Morales y A. León, Adiós a los mitos de la innovación: Una guía práctica para innovar en América Latina, San José, Costa Rica: Innovare, 2013.

[8] C. Nikulin, A. Arata, R. Stegmaier y F. Soto, «Identificación de Factores Críticos de Innovación para Modelo de Medición de Innovación por Etapas que sean representativos para los Indicadores Internacionales de I+D+i mediante la Formación de Clúster,» Sciences for Innovation, vol. 1, n. ${ }^{\circ}$, pp. 19-34, 2011. 
[9] P. A. Amar Sepúlveda y G. Angulo Cuentas, «Estudio prospectivo del sector metalmecánico en la Región Caribe Colombiana / Barranquilla,» Cámara de Comercio de Barranquilla, Barranquilla, Colombia, 2004.

[10] N. Hewitt-Dundas, «Resource and capability constraints to innovation in small and large plants», Small Business Economics, vol. 3, n. 26, pp. 257-277, 2006.

[11] C. González Campo y A. Hurtado Ayala, «Transferencia tecnológica, capital humano y cooperación: factores determinantes de los resultados innovadores en la industria manufacturera en Colombia 2007-2008,» Revista Informador Técnico, vol. 1, n. ${ }^{\circ}$ 76, pp. 32-45, 2012.

[12] L. Peeters, G. Swinnen y M. Tiri, Patterns of Innovation in the Flemish Business Sector: A Multivariate Analysis of CIS-3 Firm Level Data, IWT-Observatory, 2004.

[13] D. A. Ovallos Gazabón, «Patrones de innovación tecnológica en la industria manufacturera colombiana. El caso del sector metalmecánico de la ciudad de Barranquilla,» Universidad Tecnológica de Bolívar - Tesis de Maestría, Cartagena, Colombia, 2013.

[14] F. Becerra y C. Álvarez, «El talento humano y la innovación empresarial en el contexto de las redes empresariales: el clúster de prendas de vestir en Caldas-Colombia,» Estudios Gerenciales, vol. 27, n. ${ }^{\circ} 119$, pp. 209-234, 2011.

[15] L. C. Arraut, «La innovación de tipo Organizacional en las empresas manufactureras de Cartagena de Indias,» Semestre Económico, vol. 11, n. 22, pp. 185-203, 2009.

[16] M. Tushman y C. O’Reilly. , La organización ambidiestra, Boston, EE. UU.: Harvard Business Review, 2004.

[17] Fundación COTEC para la Innovación Tecnológica, «La innovación en sentido amplio: Un modelo empresarial. Análisis conceptual y empírico,» COTEC, Madrid, España, 2010. 